\title{
Evidence for loss of heterozygosity in human psoriatic lesions
}

\author{
G.ZACHOS, $* \dagger$ E.KOUMANTAKI, $†$ A.VARELTZIDIS $\ddagger$ AND D.A.SPANDIDOS*† \\ *Institute of Biological Research and Biotechnology, National Hellenic Research Foundation, 48 Vas. Constantinou Ave, \\ Athens 11635, Greece \\ †Division of Virology, M edical School, University of Crete, Heraklion, 71409, Greece \\ $\ddagger$ M edical School, University of Athens, Department of Dermatology, 'A. Sygros' Hospital, Athens, Greece
}

Accepted for publication 30 April 1998

Summary Psoriasis, a disease of human skin, is characterized by abnormal differentiation and hyperproliferation of keratinocytes; it has a genetic background. Using 11 highly polymorphic microsatellite markers on eight chromosome arms, we performed an allelotype analysis in 14 psoriatic plaques, in order to reveal any chromosome deletions involved in the development of the disease. We detected loss of heter ozygosity ( $\mathrm{LOH}$ ) on at least one microsatellite marker in nine of $14(64 \%)$ cases. We also observed particular genetic loci altered with $\mathrm{LOH}$, on chromosomes $3 p, 7 p / q$ and $8 p$. Our results suggest that LOH is an important phenomenon in the development of psoriatic plaques, providing evidence for deletion of regulatory genes.

Psoriasis affects approximately $2-3 \%$ of the European population and presents with red scaly patches on the skin. The major pathogenic features of psoriasis include abnormalities in differentiation, hyperproliferation of keratinocytes and infiltration of inflammatory components into the skin. ${ }^{1}$ Epidemiological data and familial association suggest a hereditary base for transmission of psoriasis; however, it does not appear to follow simple autosomal dominant or recessive patterns. This may be due to multifactorial inheritance or to inheritance only of a predisposition to disease that requires an environmental stimulus for expression, such as stress, drugs, viral infection or dietary factors. ${ }^{2-5}$

The molecular genetic basis of the disorder was investigated. Evidence that a locus for familial psoriasis susceptibility maps to the distal end of chromosome $17 \mathrm{q}$ was reported; however, it proved to be genetically heterogeneous and is possibly involved in familial psoriasis under a polygenic model. ${ }^{6,7}$ Other potent genes for familial psoriasis were mapped to chromosome $6 \mathrm{p} 21$, within the major histocompatibility complex (MHC) and close to the class 1 human leucocyte associated antigen (HLA) loci, and to chromosome $4 q^{8,9}$ Altered expression of proto-oncogenes, which are regulators of the cell cycle, was proposed as a mechanism of hyperproliferation and abnormal differentiation of keratinocytes. However, the proto-oncogenes tested (c-fos, c-jun, c-H-ras, c-erbB2, cmyc, c-raf) were not implicated in the pathogenesis of

Correspondence: Professor D.A.Spandidos.

Present address: G.Zachos, Institute of Virology, University of Glasgow, Church St, Glasgow G11 5JR, U.K. psoriatic epidermal hyperplasia, either with quantitative, or qualitative changes. ${ }^{10-12} \mathrm{~A}$ role in the regulation of epidermal proliferation for genes on chromosome 1q21, which are co-ordinately overexpressed in psoriatic lesions, was proposed; aberrantly regulated genes in diseased skin included mitochondrial genes, junction proteins and protease inhibitors. ${ }^{13,14}$

The aim of our investigation was to perform an allelotype analysis in 14 psoriatic plaques, in order to reveal any chromosome deletions involved in the development of the disease. This analysis could provide information about the inactivation of regulatory genes. Such an inactivation might result in hyperproliferation and abnormal differentiation of psoriatic cells, a model analogous to the inactivation of tumour suppressor genes in cancer development, thus also extended to non-cancerous lesions. ${ }^{15,16}$ We performed loss of heterozygosity (LOH) analysis of eight chromosome arms using 11 highly polymorphic microsatellite markers. Our results suggest that $\mathrm{LOH}$ is a detectable phenomenon in psoriatic plaques and contributes to the molecular background of the disease.

\section{Materials and methods}

Biological material and DNA extraction

Fourteen specimens (keratinocytes from punch biopsies of patients with psoriasis) were obtained from the Department of Dermatology, 'A.Sygros' Hospital, Athens. Biopsies confirmed the histopathological phenotype of 
Table 1. Loss of Heterozygosity analysis in human psoriatic lesions

\begin{tabular}{ccccccccccccc}
\hline Patient no. & D2S138 & D2S147 & D3S1478 & D3S1298 & FGA & D7S478 & D7S519 & ANK1 & D8S7 & D9S290 & D21S1245 \\
\hline 1 & - & - & + & - & - & - & - & - & - & - & - \\
2 & - & - & - & + & - & + & + & + & + & - & - \\
3 & - & - & - & - & - & - & - & - & - & - & - \\
4 & - & - & - & + & - & + & + & + & + & - & - \\
5 & - & - & - & - & - & - & - & - & - & - & - \\
6 & - & - & + & + & - & + & - & + & + & - & - \\
7 & - & - & + & + & - & - & - & - & + & - & - \\
8 & - & - & - & - & - & - & - & - & - & - & - \\
9 & - & - & - & - & - & - & - & - & - & - & - \\
10 & - & - & - & + & - & - & - & - & + & - & - \\
11 & - & - & - & - & - & - & - & - & - & - & - \\
12 & - & - & - & - & - & - & - & - & + & - & - \\
13 & - & - & + & - & - & - & - & - & - & - & - \\
14 & - & - & + & - & - & - & + & - & + & - & - \\
\hline
\end{tabular}

psoriasis. Ten patients were females and four males. Twelve patients (numbers 1-12) had a family history of the disease and/or an onset below the age of 40 . Patients 13 and 14 had a late onset of the disease and no family history. Matched blood samples were used as negative controls. DNA was extracted from psoriasis plaque specimens and blood samples using standard methods. $^{15}$

\section{M icrosatellite analysis}

Eleven microsatellite markers were used (Table 1). ${ }^{17}$ Markers D2S138 and D2S147 are located on chromosomes 2q21-q33 and 2p14-q13, respectively. Markers D3S1478 and D3S1298 are located on chromosomes 3p21.3-p21.2 and 3p24.2-p22, within the hMlh1 gene. FGA lies on chromosome 4q28. Markers D7S478 and D7S519 are located on chromosome 7p15-q22, within the Pms2 gene. ANK1 and D8S7 lie on chromosome regions 8p21.1-p11.2 and 8p23, respectively. Finally, markers D9S290 and D21S1245 are located on chromosomes 9q32-q34.1 and 21q22.2- qter, respectively.

PCR reactions were performed in a $50 \mu \mathrm{L}$ reaction volume, containing $100 \mathrm{ng}$ of genomic DNA, $200 \mu \mathrm{mol} / \mathrm{L}$ dNTPs, $10 \mathrm{pmol}$ of each forward and reverse primer, $10 \mathrm{mmol} / \mathrm{L}$ Tris- $\mathrm{HCl}, \mathrm{pH} 8.8,1.5 \mathrm{mmol} / \mathrm{L} \mathrm{MgCl}_{2}$, $50 \mathrm{mmol} / \mathrm{L} \mathrm{KCl}, 0 \cdot 1 \%$ Triton X-100 and $2 \mathrm{U}$ Dynazyme ${ }^{\mathrm{TM}}$ II DNA polymerase (Finnzymes OY, Espoo, Finland). The reactions were denatured at $98^{\circ} \mathrm{C}$ for $3 \mathrm{~min}$, prior to the addition of the polymerase, and the DNA was subsequently amplified for 30 cycles, at $94{ }^{\circ} \mathrm{C}, 54-58^{\circ} \mathrm{C}$,
Case No.

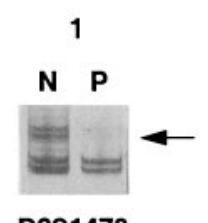

D3S1478

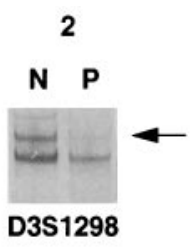

D3S1298

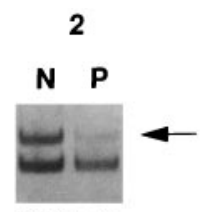

D7S478

Case No.
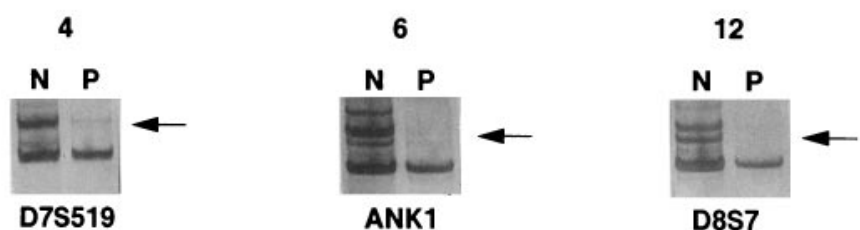

D8S7 
$72{ }^{\circ} \mathrm{C}$ each step. Ten microlitres of the PCR product were electrophoresed in a $10 \%$ polyacrylamide gel and stained with silver. LOH was scored when a more than $70 \%$ reduction in the intensity of one allele in the heterozygous specimens was observed in DNA from psoriasis plaques, compared with the corresponding blood samples. The remaining faint bands of the lost allele could be due to contamination with non-lesional skin. The analysis in the $\mathrm{LOH}$ positive cases was repeated at least twice and the results were highly reproducible.

\section{Results}

Nine of $14(64 \%)$ cases tested exhibited LOH on at least one microsatellite marker (Table 1, Fig. 1). Allelic imbalance was observed for the locus 3p24-p21.2 (markers D3S1478, D3S1298) in eight of $14(57 \%)$ cases, for the locus 7p15-q22 (markers D7S478, D7S519) in four of $14(28 \%)$ cases, and for the region 8p21.1-p11.2 and 8p23, encompassing almost the entire $8 p$ chromosome arm (markers ANK1, D8S7), in seven of $14(50 \%)$ cases. Retention of heterozygosity was observed for the remaining chromosome loci tested, in all cases.

\section{Discussion}

Little is still known about the genetic background of the disorder. We therefore tested for LOH genetic loci dispersed in multiple chromosome regions. Furthermore, possible candidates like the mismatch repair genes hMIh1 and Pms2, the VHL onco-suppressor and the $4 q$ region, previously linked to psoriasis, were included in our study. The incidence of $\mathrm{LOH}$ was investigated on eight chromosome arms (2p, 2q, 3p, 4q, 7p/q,8p, 9q, 21q) using 11 microsatellite markers. Loss of heterozygosity of particular chromosome regions was reported in the development of human neoplasia, indicating the presence of onco-suppressor genes. ${ }^{18}$ Our experimental results suggest that LOH represents an important phenomenon in the development of human psoriatic lesions as well, as the majority of the patients (nine of $14,64 \%$ ) exhibited $\mathrm{LOH}$ on at least one microsatellite marker. Moreover, allelic imbalance was observed for regions on the chromosome arms $3 p$ (eight of 14, 57\%), $7 p / q$ (four of $14,28 \%$ ) and $8 p$ (seven of $14,50 \%$ ). This suggests that genes important for suppressing cell cycle alterations are located in these particular chromosome regions; the mechanism may be similar to that seen in neoplastic disorders.

The region in chromosome $3 p$ tested in our study, is deleted in many solid tumours and is considered as the location of a lung cancer suppressor gene ${ }^{19}$ and of the VHL onco-suppressor, which is implicated in renal cell carcinomas and haemangioblastomas. ${ }^{20}$ In addition, allele loss of the hMlh1 mismatch repair gene located in this region, is implicated in human cancers exhibiting high frequency of somatic mutations. ${ }^{21}$ Moreover, allelic loss on chromosome $7 q$ is observed in human tumours. ${ }^{22}$ The Pms2 mismatch repair gene is located in this region and is a likely candidate for the allelic imbalance that we detected. ${ }^{23}$ Furthermore, a frequently deleted region on chromosome $8 p$, overlapping the locus tested in our study, was established in studies of colorectal and bladder tumours. ${ }^{24}$

In conclusion, we identified chromosome loci where genes regulating cell differentiation, growth and proliferation may be located, and suggest that $\mathrm{LOH}$ contributes to the development of human psoriatic lesions. To our knowledge, this is the first report of $\mathrm{LOH}$ in a chronic inflammatory disease with a genetic background. Extension of our observations in more chromosome arms, as well as a detailed mapping of the deleted regions using additional sets of microsatellite markers, may be useful in understanding the molecular basis of the disorder.

\section{References}

1 Ortonne J-P. A etiology and pathogenesis of psoriasis. $\mathrm{Br}$ J Dermatol 1996; 135 (Suppl. 49): 1-5.

2 Harvima RJ, Viinamaki H, Harvima IT et al. Association of psychic stress with clinical severity and symptoms of psoriatic patients. Acta Derm Venereol (Stockh) 1996; 76: 467-71.

3 Gold MH, Holy AK, Roenigk HH. Beta-blocking drugs and psoriasis. J Am Acad Dermatol 1988; 19: 837-41.

4 Coopman SA, Johnson TM, Platt R et al. Cutaneous disease and drug reactions in HIV infection. N Engl J M ed 1993; 328: 1670-4.

5 Naldi L. Psoriasis. Dermatol Clin 1993; 13: 635-47.

6 TomfohrdeJ, Silverman A, Barnes R et al. Gene to familial psoriasis susceptibility mapped to the distal end of human chromosome 17q. Science 1994; 264: 1141-5.

7 Nair RP, Guo SW, Jenisch S et al. Scanning chromosome 17 for psoriasis susceptibility: lack of evidence for a distal $17 q$ locus. Hum Hered 1995; 45: 219-30.

8 Trembath RC, Clough RL, Rosbotham JL et al. Identification of a major susceptibility locus on chromosome $6 p$ and evidence for further disease loci revealed by a two stage genome-wide search in psoriasis. Hum M ol Genet 1997; 6: 813-20.

9 Matthews D, Fry L, Powles A et al. Evidence that a locus for familial psoriasis maps to chromosome 4q. Nat Genet 1996; 14: 231-3.

10 Basset-Seguin N, Escot C, Moles JP et al. c-fos and c-jun protooncogene expression is decreased in psoriasis: an in situ quantitative analysis. J Invest Dermatol 1991; 97: 672-8.

11 Elder JT, Klein SB, Tavakkol A et al. Growth factor and protooncogene expression in psoriasis. J Invest Dermatol 1990; 95: 79S. 
12 Takahashi $\mathrm{H}$, lizuka $\mathrm{H}$, Katagiri M. No evidence for the mutation of ras gene in psoriatic epidermis. Arch Dermatol Res 1990; 282: 811.

13 Hardas $\mathrm{BD}$, Zhao $\mathrm{X}$, Zhang $\mathrm{J}$ et al. Assignment of psoriasin to human chromosomal band 1q21: coordinate overexpression of clustered genes in psoriasis. J Invest Dermatol 1996; 106: 753-8.

14 Rivas MV, Jarvis ED, Morisaki S et al. Identification of aberrantly regulated genes in diseased skin using the cDNA differential display technique. J Invest Dermatol 1997; 108: 188-94.

15 Hatzistamou J, Kiaris H, Ergazaki M, Spandidos DA. Loss of heterozygosity and microsatellite instability in human atherosclerotic plaques. Biochem Biophys Res Commun 1996; 225: 186-90.

16 Spandidos DA, Sourvinos G, Kiaris H, Tsamparlakis J. Microsatellite instability and loss of heterozygosity in human pterygia. $\mathrm{Br} J$ Ophthalmol 1997; 81: 493-6.

17 Murray JC, Buettow KH, Weber JL et al. A comprehensive human linkage map with centimorgan density. Cooperative Human Linkage Center (CHLC). Science 1994; 265: 2049-54.

18 Lasco D, Cavenee W, Nordenskjold M. Loss of constitutional heterozygosity in human cancer. Annu Rev Genet 1991; 25: 281-314.
19 Wei MH, Latif F, Bader S et al. Construction of a 600-kilobase cosmid clone and generation of a transcriptional map surrounding the lung cancer tumor suppressor gene (TSG) locus on chromosome 3 p21.3: progress toward the isolation of a lung cancer TSG. Cancer Res 1996; 56: 1487-92.

20 Prowse AH, Webster AR, Richards FM et al. Somatic inactivation of the VHL gene in Von Hippel-Lindau disease tumors. Am J Hum Genet 1997; 60: 765-71.

21 Thibodeau SN, French AJ, Roche PC et al. Altered expression of $\mathrm{hMSH} 2$ and $\mathrm{hMLH} 1$ in tumors with microsatellite instability and genetic alterations in mismatch repair genes. Cancer Res 1996; 56: 4836- 40.

22 Kerr J, Leary JA, Hurst T et al. Allelic loss on chromosome 7q in ovarian adenocarcinomas: two critical regions and a rearrangement of the PLANH1 locus. Oncogene 1996; 13: 1815-18.

23 Narayanan L, Fritzell JA, Baker SM et al. Elevated levels of mutation in multiple tissues of mice deficient in the DNA mismatch repair gene Pms2. Proc Natl Acad Sci USA 1997; 94: 3122 7.

24 Macoska JA, Trybus TM, Benson PD et al. Evidence for three tumor suppressor gene loci on chromosome $8 p$ in human prostate cancer. Cancer Res 1995; 55: 5390-5. 